\title{
Importance and Methods of Residual Stress Profile Measurement
}

Zdeněk Pitrmuc, Vítězslav Rázek, Libor Beránek, Marin Vrabec, Jan Šimota

Department of Machining, Process Planning and Metrology, Faculty of Mechanical Engineering, Czech Technical University in Prague, Technická 4, 16607 Praha 6, E-mail: zdenek.pitrmuc@fs.cvut.cz; vitezslav.razek@fs.cvut.cz, libor.beranek@fs.cvut.cz; martin.vrabec@fs.cvut.cz jan.simota@fs.cvut.cz;

The contribution is dedicated to surface integrity assessment of components from the point of view of residual stress profile after machining and finishing technologies. Residual stresses play the key role for dynamic life and service reliability of the part, especially rotating aircraft airfoils made of titanium and nickel base alloys. Except a brief summary of measurement methods practical experience with application of Beam deflection method combined with electrolytic etching is published. Specific measurement results for real aircraft Ti6Al4V airfoils and Ti6Al4V plates following its manufacturing technology are the subject of experimental part.

Keywords: measurement of residual stress profile; beam deflection method; electrolytic etching; titanium airfoils, thumbling; laserpeening

\section{Acknowledgement}

This work was supported by the governmental funding of Technological Agency of Czech Republic-project number TH01011405.

\section{References}

[1] MÁDL, J., RÁZEK, V., KOUTNÝ, V., KAFKA, J. (2011). Surface Integrity in Notches Machining. In: Precision Machining. Uetikon-Zurich: Trans Tech Publications, p. 176-181. ISBN 978-3-03785-297-2.

[2] LI, YU-JIA, FU-ZHEN XUAN, ZHENG-DONG WANG A SHAN-TUNG TU. (2010). Effects of Residual Stresses on the High Cycle Fatigue Behavior of Ti-6Al-4V. In: ASME 2010 Pressure Vessels and Piping Conference: Volume 5 [online]. 2010 [cit. 2015-02-27]. DOI: 10.1115/pvp2010-25364.

[3] KAFKA, J., RÁZEK, V. (2011). Vliv technologických procesů na zbytková pnutí v povrchové vrstvě. Technická univerzita Liberec.

[4] YAKOVlEV, M. G., V. A. GORELOV, N. S. MERKUlOVA, A. S. KUDROV. (2014). Study of the influence of residual stresses on the fatigue strength of samples made of titanium and nickel alloys. Journal of Machinery Manufacture and Reliability [online]. vol. 43, issue 5, s. 389-392 [cit. 2015-02-24]. DOI: 10.3103/s1052618814050203.

[5] SCHAJER, G. Practical residual stress measurement methods. 1 online resource (330 pages). ISBN 9781118402818-.

[6] RÁZEK, V., KOUTNÝ, V., MÁDL, J. (2009). Řezný proces a kvalita obrobené plochy při progresivním obrábění, TRANSFER 2009

[7] NECKÁŘ, F., KVASNIČKA, I. (1991). Vybrané statě z úběru materiálu. Vyd. 1. Praha: Ediční stř̌edisko ČVUT, 1991, 88 s. ISBN 80-010-0696-4.

[8] KAFKA, J. aj. (1982). Vznik zbytkových pnutí ve strojních součástech a metodika jejich měření [Zpráva o výzkumu]. Praha: ČVUT Fakulta Strojní.

[9] GAUTHIER, J., T.W. KRAUSE a D.L. ATHERTON. (1998). Measurement of residual stress in steel using the magnetic Barkhausen noise technique. NDT [online]. 1998, vol. 31, issue 1, s. 23-31 [cit. 2013-11-27]. DOI: 10.1016/S0963-8695(97)00023-6. Dostupné z: http://linkinghub.elsevier.com/retrieve/pii/S0963869597000236

[10] KÖHLER, J., T. GROVE, O. MAIß a B. DENKENA. (2012). Residual Stresses in Milled Titanium Parts. Procedia CIRP [online]. vol. 2, s. 79-82 [cit. 2015-02-25]. DOI: 10.1016/j.procir.2012.05.044.

[11] WONG, W., M. R. HILL. (2012). Superposition and Destructive Residual Stress Measurements. Experimental Mechanics [online], vol. 53, issue 3, s. 339-344 [cit. 2015-02-25]. DOI: 10.1007/s11340-012-9636-y.

[12] MÁDL, J., RÁZEK, V., KOUTNÝ, V., KAFKA, J. (2014). Surface Integrity in Notches Machining. Manufacturing Technology. 2014, 13(2), 188-193. ISSN 1213-2489. 